\title{
La formación de futuros docentes: nuevas habilidades en entornos cambiantes

\author{
Initial training of future teachers: new skills in changing environments
}

\author{
Guadalupe Trigueros Gordillo ${ }^{1}$, Cristina Ceballos Hernández ${ }^{2}$ \\ trigueros@us.es, cceballos@us.es
}

\author{
${ }^{1}$ Dpto. Teoría e Historia de la Educación y Pedagogía Social \\ Universidad de Sevilla \\ Sevilla, España
}

\author{
${ }^{2}$ Dpto. Economía Financiera y Dirección de Operaciones \\ Universidad de Sevilla \\ Sevilla, España
}

\begin{abstract}
Resumen- En este trabajo se plantean algunas reflexiones acerca de la formación inicial del profesorado de secundaria, especialmente tras los últimos acontecimientos derivados de la pandemia. Detectadas algunas necesidades en los profesores en formación (PF), se presenta un amplio plan llevado a cabo, basado en la orientación del futuro docente, la enseñanza en herramientas digitales y entornos online, así como habilidades comunicativas. Mediante procesos de encuestación, se recoge la percepción de los PF. Estos profesores valoran muy positivamente las acciones desarrolladas, tanto en lo que han supuesto para su incorporación a las prácticas (en muchos casos en entornos online) como en lo que respecta a la mejora percibida en sus habilidades de comunicación tanto en entornos presenciales como online. Las acciones sobre la orientación laboral suponen, por un lado, un claro acercamiento a los centros educativos y sus problemáticas, y por otro, las jornadas de oposiciones tiene efectos significativos en los conocimientos sobre este proceso, incrementan la intención de presentarse a oposiciones por parte de los PF y aumentan la confianza de superar con éxito este proceso.
\end{abstract}

Palabras clave: Formación del profesorado; Enseñanza Secundaria, Bachillerato y FP; orientación laboral; competencias

Abstract- In this paper some reflections are presented about the initial training of secondary school teachers, especially after the pandemic. Detected some needs in teachers in training (FP), a comprehensive plan is presented. It is based on the orientation of the future teacher, teaching in digital tools and online environments, as well as communication skills. Through survey processes, the perception of the FP is collected. These teachers value the actions carried out very positively: important regarding their incorporation into the online practices and in relation to the perceived improvement in their communication skills (face-to-face and online environments). The actions on career orientation suppose a good approach to the educational centers and they have significant effects on the incorporation into the educational public centers (knowledge about this process, intention and confidence).

Keywords: Teacher training; secondary high school; vocational training; career orientation; competences

\section{INTRODUCCIÓN}

El Covid-19 se ha convertido en un catalizador para que las instituciones educativas, con ritmos de cambios lentos, y acomodadas en la metodología, hayan buscado soluciones innovadoras en un período de tiempo relativamente corto. La pandemia del Covid-19 ha puesto en crisis las habilidades de muchos docentes en todos los niveles educativos así como los recursos de los centros, en un momento en el que el principal objetivo ha sido dar continuidad a la vida académica. Este reto de adaptación vertiginosa no siempre ha tenido traído consigo los resultados más deseables en términos de rendimiento académico, adquisición de conocimientos, motivación, etc. La improvisación en la educación a distancia ha evidenciado también debilidades, poniéndose de manifiesto una falta de formación de los docentes en activo en el uso de TIC y metodologías para promover la autonomía (Manzano-Sánchez, Valero Valenzuela y Hortigüela-Alcalá, 2021; Martínez-Garcés y Garcés-Fuenmayor, 2020; Molina-Pérez y Pulido-Montes, 2021). Se ha observado un déficit en la formación y los recursos, que ha dificultado la digitalización de los procesos de enseñanza-aprendizaje. En algunas ocasiones las videoconferencias, además, no han conseguido captar la atención del alumnado reduciéndose su motivación, concluyéndose la necesidad de que el profesorado esté formado en la modalidad online y en los recursos que este tipo de enseñanza ofrece (Cifuentes-Faura, 2020). Trabajar en la adquisición de competencias tecnológicas favorecerá la cercanía con los estudiantes en el marco de un aprendizaje a distancia (Ramos-Huenteo, García-Vásquez, Olea-González, Lobos-Peña y Sáez-Delgado, 2020). La conveniencia de impartición de breves cursos de formación para docentes en el uso efectivo de las plataformas digitales se señalaba ya en el Plan educativo de emergencia propuesto para finales de 2020 en el informe elaborado por FEDEA (Núñez et al., 2020).

Las investigaciones muestran además que la formación del profesorado en las innovaciones tecnológicas es demasiado lenta, y no consigue ofrecerles la competencia digital necesaria (Elstad y Christophersen, 2017; Instefjord y Munthe, 2017, citados en Engen, 2019). La modificación del programa que habilita para el ejercicio de la docencia en secundaria es una de las mejoras que se propone en el informe de Núñez et al. (2020), siendo necesario reforzar con carácter de urgencia la formación de profesores en competencias digitales y métodos de aprendizaje online. Pero también hay otras habilidades que son incluso más necesarias de poseer y gestionar para que la enseñanza online sea eficiente y efectiva. En este sentido podemos señalar, entre otras: la capacidad de organización de los contenidos; de administración de tiempos; de comunicación online; habilidades y conocimientos técnicos y tecnológicos, o habilidades pedagógicas en entornos digitales, para poder gestionar, administrar y enseñar los contenidos a través de una plataforma educativa online. Este necesario enfoque en la 
preparación de los profesores en formación de secundaria (en adelante PF) debe completarse con una adecuada orientación hacia la profesión docente. En los últimos meses se ha acelerado el proceso de incorporación de profesores a los centros educativos, como respuesta a las necesidades derivadas de la pandemia y como consecuencia del envejecimiento de las plantillas. Muestra de ello es el aumento del número de plazas de oposiciones convocadas en nuestro país.

Derivado de lo expuesto anteriormente, en el Máster de Formación del Profesorado de la Universidad de Sevilla, donde se prepara a los futuros docentes de secundaria, bachillerato y $\mathrm{FP}$, se ha diseñado un plan de formación complementario que ayude a la adquisición de determinadas habilidades y permita a los PF responder más eficazmente a las necesidades derivadas del nuevo entorno. El objetivo ha sido mejorar la formación de los PF, por lo que en este trabajo se presentan estas acciones y se analiza la percepción de estos. En la siguiente sección se describe el contexto en el que se ha desarrollado el trabajo, resaltándose las características que hacen que el mismo sea transferible a otros casos. Posteriormente, se presenta el plan de refuerzo formativo frente a la pandemia para los $\mathrm{PF}$, valorando los resultados de las principales acciones. Finalizamos con una discusión de los hallazgos y con las conclusiones del trabajo.

\section{CONTEXTO}

El Máster de Formación del Profesorado tiene como misión habilitar para el ejercicio de la profesión, a la vez que constituye la base de la formación inicial del profesorado. Los conocimientos y las competencias adquiridas en esa fase no constituyen una base única, estanca, inamovible, suficiente y definitiva, pero sí son un componente imprescindible cuya calidad ha de ser garantizada para el adecuado acceso a la profesión docente. Según el estudio de López y García (2021) sobre el profesorado de educación secundaria, basado en el informe PISA 2018, existe una desviación notable entre lo que ha sido la formación inicial, según la percepción del profesorado en ejercicio, y lo que debería ser, según la apreciación de la OCDE. Más de una tercera parte considera que su formación para la profesión no incluyó lo que puede ser considerado como esencial para enseñar.

En general, los PF apenas tienen conocimientos relacionados con la práctica docente cuando inician el Máster. Estudios realizados en diversas universidades concluyen que los futuros docentes perciben un incremento en su desarrollo competencial tras cursarlo. Se observa una mejora más significativa en los más recientes, entendiéndose esto como proceso de optimización de la formación a medida que se adquiere experiencia en el Máster (Manso y Martín, 2014; Serrano y Pontes, 2015; Sarceda-Gorgoso, Santos González y RegoAgraso, 2020). Asimismo, se evidencian diferencias estadísticamente significativas entre las universidades participantes (Sarceda-Gorgoso, et al., 2020). Por otro lado, no todos aquellos que optan por cursar el Máster tienen vocación docente. Muñoz-Fernández, Rodríguez-Gutiérrez, y LuqueVílchez (2019) identifican, según sus motivaciones, tres grupos o tipologías de PF. Los que consideran que la docencia es una mera salida profesional (vocación circunstancial o sin vocación, 24,6\%), los que descubren satisfacción al cursar el Máster (vocación encontrada, 33,6\%) y los que manifiestan vocación intrínsecamente docente $(41,8 \%)$. Las diversas motivaciones deben de ser tenidas en cuenta para la selección, elaboración y planificación de la formación inicial que recibirá el futuro profesorado, de forma que se diseñen espacios de desarrollo y fomento de su dimensión vocacional. Asimismo, puede resultar interesante trabajar sobre la base de las motivaciones, actitudes y expectativas de los candidatos a profesores (MuñozFernández et al., 2019). En otras palabras, la orientación docente debe jugar en el Máster un papel clave. El Máster es ofertado en España por 46 universidades públicas y 19 privadas. Más de $450 \mathrm{PF}, 16$ especialidades distintas, más de 225 profesores, alrededor de 80 departamentos implicados en la docencia y 4 convenios de doble máster dan una idea de su dimensión e importancia en la Universidad de Sevilla, donde se contextualiza este estudio.

\section{DESCRIPCIÓN}

Teniendo como objetivo las competencias que deben de adquirir los futuros docentes, se han puesto en marcha en los últimos cursos académicos distintas acciones formativas complementarias a los contenidos que reciben los PF. Aunque el plan es mucho más amplio, este trabajo se centra en las acciones más relevantes desde el punto de vista de su mejora competencial como docentes en el contexto actual. Estas acciones se estructuran en tres grandes bloques: orientación a la labor docente, adquisición de habilidades de comunicación en entornos presenciales y online y acciones encaminadas a la adquisición de habilidades en el uso de las TIC (figura 1).

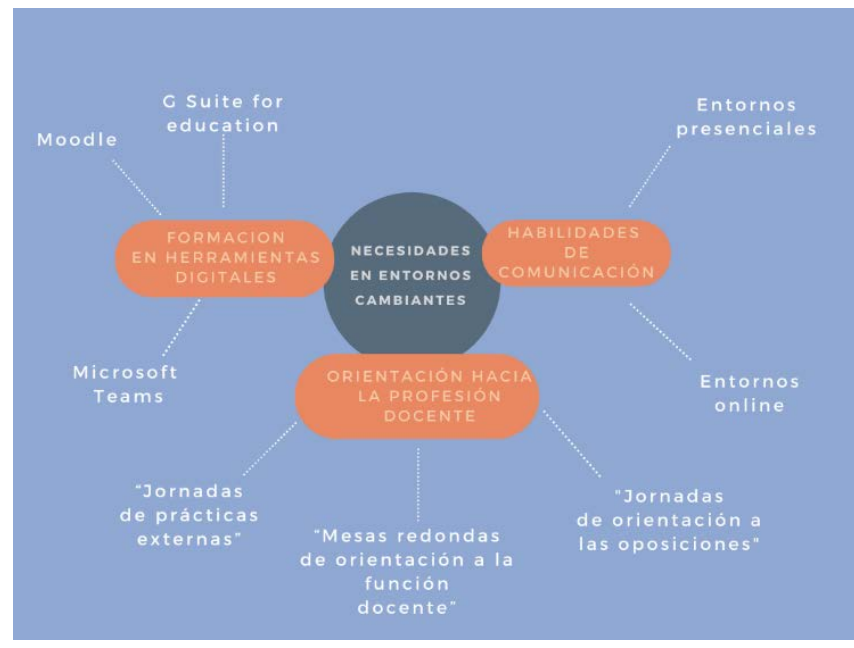

Figura 1. Plan de formación complementario para PF

\section{A. Acciones encaminadas a la orientación hacia la profesión docente}

El primer bloque de acciones está enfocado a la orientación de los futuros docentes. En el máster de la Universidad de Sevilla consideramos fundamental la colaboración con los centros de secundaria, que deben ser referentes en el diseño de la formación inicial del profesorado. Por ello anualmente se organizan "Mesas redondas de orientación a la función docente", "Jornadas de prácticas externas", y "Jornadas de orientación a las oposiciones". Las primeras, celebradas a principio de curso, se diseñan atendiendo a las 16 especialidades ofertadas, habida cuenta de las diferencias entre ellas por materias. Los profesores de secundaria invitados tratan diversas cuestiones acerca de su experiencia y su labor profesional en la enseñanza, dando paso a un debate en el que participan los PF. Se propicia así un diálogo que invita a reflexionar sobre la salida profesional (centros públicos, concertados, privados), las competencias para un desempeño 
exitoso de la función docente, recomendaciones, funcionamiento de los centros educativos, aprovechamiento del período de prácticas, formación adicional recomendable, etc. Las "Jornadas de prácticas externas" tienen como objetivo presentar a los PF experiencias y buenas prácticas desarrolladas con éxito en los centros educativos. Están relacionadas con metodologías docentes, sistemas de evaluación, innovaciones educativas, atención a la diversidad, organización en centros educativos, sistemas de FP dual, entre otros, estando a cargo de profesores de secundaria en activo. Por último, el PF demanda una orientación para afrontar la preparación de oposiciones docentes de acceso a la función pública, habida cuenta de la preocupación y desconocimiento que le genera todo lo relacionado con este proceso. Participan profesores en activo con amplia experiencia, bien como miembros en tribunales de oposición y/o como preparadores. Se dividen en 5 sesiones: estructura de la oposición (S1), baremación de currículum (S2), claves y consejos del tribunal y preparadores (S3), experiencia de opositores recientes (S4) y claves para una buena defensa (S5). La segunda edición de estas jornadas, que se ha desarrollado online debido a las circunstancias sanitarias, ha acogido a más de 350 PF. Debido a las diferencias por especialidades en los ejercicios de la oposición, se realizan sesiones de forma paralela para cada especialidad. Esto ha llevado a contar con la participación de 24 ponentes en esta última edición.

\section{B. Capacitación en habilidades de comunicación}

El futuro docente deberá ser un educador implicado en la formación integral del alumno, tanto desde el punto de vista personal como social. Ello requiere una sólida formación sociológica y psicopedagógica, dominio de destrezas sociales y de interacción, así como una idónea competencia comunicativa. En la formación de los PF se trabajan las habilidades comunicativas como parte de las competencias transversales que deben adquirirse. Como se recoge en el programa de los talleres del Master ${ }^{1}$, las técnicas de comunicación constituyen uno de los pilares fundamentales para el éxito de la labor docente y son necesarias para:

- Comunicar el mensaje, facilitando así la comprensión del mismo por parte del alumno.

- Estructurar la información adecuadamente, estableciendo un hilo conductor que permita a los alumnos asimilar los conocimientos de forma correcta.

- Tener en cuenta aspectos de comunicación verbal y no verbal para facilitar la comprensión del mensaje al alumno.

- Tener un especial cuidado de la voz, al ser ésta la herramienta de trabajo.

- Saber gestionar el estrés que cualquier exposición en público produce.

Sin embargo, diversos estudios han puesto de manifiesto la carencia de los docentes noveles en lo referido a habilidades y estrategias para comunicar y comunicarse con los estudiantes cuando se adentran en el ejercicio de la enseñanza (Camus, Iglesias y Lozano, 2019). La principal consecuencia de esto es que no se genera un espacio comunicativo funcional para el aprendizaje, por lo que señalan la necesidad de establecer líneas de trabajo al respecto en la formación inicial del profesorado.
Por este motivo el plan incluye un ciclo de talleres para la mejora de las habilidades comunicativas, tanto en entornos presenciales como online. El objetivo es ofrecer a los PF técnicas eficaces de comunicación que les permitan conectar mejor con sus alumnos y les ayuden a impartir su docencia, comenzando por el período de prácticas en los centros de secundaria. Son talleres prácticos de 8 horas de duración, en los que se realizan simulaciones, presentaciones reales y exposiciones, recibiendo el futuro docente un feedback individualizado en el que se identifican puntos fuertes y aspectos de mejora.

\section{Capacitación en herramientas docentes digitales}

Con la llegada del Covid-19 el uso de herramientas virtuales o campus online ha pasado de ser un recurso para mejorar el rendimiento a convertirse en una necesidad. En bastantes casos, el profesorado se ha expuesto por primera vez a ellas, y ha debido de aprender rápidamente no sólo su uso, sino también una nueva jerga y su relación con metodologías activas con el fin de involucrar a los estudiantes en actividades interactivas y colaborativas. Aunque en ocasiones se ha recurrido al autoaprendizaje, en muchas otras el aprendizaje ha surgido por apoyo entre compañeros. Como señala Donitsa-Schmidt y Ramot (2020) han sido significativas las docenas de comunidades de aprendizaje profesional (PLC) de abajo hacia arriba que emanaron de los propios docentes, observándose una mayor colaboración entre pares. Esto justifica la necesidad de acciones formativas, desde los centros y en la formación inicial de los docentes. Para mejorar la competencia digital de los PF se han puesto en marcha diversas iniciativas desde los másteres de formación del profesorado, como la recogida en RomeroGarcía, Buzón-García y de Paz-Lugo (2020). Estos autores destacan la conveniencia de formar en este sentido a los futuros profesores para poder integrar las TIC en el plan de estudios y en la práctica educativa, así como para que los estudiantes de secundaria sean capaces de adquirir a su vez dicha competencia.

Partiendo de estas premisas, el Máster ha diseñado una formación teórico-práctica para los $\mathrm{PF}$ sobre distintas herramientas digitales especialmente importantes para un correcto seguimiento online del alumnado, poniendo énfasis en la adquisición de competencias en estos entornos (diseñar contenidos y actividades, evaluar, tutorizar, fomentar el trabajo cooperativo, etc). El objetivo propuesto ha sido que el futuro docente sea capaz optimizar los recursos virtuales que encuentre en cada centro educativo. Los contenidos de esta formación online se han estructurado en tres módulos: Moodle y herramientas de creación de contenido eLearning, paquete GSuite y Microsoft Teams. En cada uno de ellos se han incluido presentaciones, videos, ejercicios, cuestionarios de autoevaluación y evaluación, etc., que se han ido liberando al ritmo de cada alumno. Se han establecido foros de discusión, mensajes en la plataforma y correo electrónico para la resolución de dudas. Para completar la acción tutorial, se han celebrado videoconferencias sobre contenidos concretos. Esta acción formativa ha concluido con la entrega de un completo proyecto en cada una de estas herramientas. Dicha formación dotó a los PF en pleno confinamiento del curso 2019/20, de conocimientos adecuados para desarrollar de forma más eficaz y eficiente el trabajo colaborativo con el tutor de secundaria una vez las prácticas se reanudaron online. La continuidad en el

\footnotetext{
${ }^{1}$ https://masteroficial.us.es/maes/sites/masteroficial.us.es.maes/files/habilidade s_de_comunicacion_para_la_labor_docente.pdf
} 
empleo de estos entornos ha hecho que se incluyera de nuevo esta formación complementaria en el 2020/21. Estas herramientas se han utilizado con mayor profundidad en el plan alternativo de prácticas diseñado para los PF que se encuentran en situación de especial riesgo y vulnerabilidad y que no han realizado prácticas de forma presencial. En esta línea, estas plataformas Learning Management System (LMS), como por ejemplo Moodle, se consideran fundamentales en la innovación educativa, pues se entienden como facilitadoras del éxito en la implantación de innovaciones metodológicas (evaluación por evidencias, aprendizaje personalizado o Flip Teaching, entre otras) (Fidalgo-Blanco, Sein-Echaluce y García-Peñalvo, 2019).

\section{Metodología}

Para la recopilación de datos se hizo uso de diversos cuestionarios en línea:

- Sobre la capacitación en "Herramientas docentes digitales", en junio de 2020 (329 respuestas, 93,5\%) y abril de 2021 (79 respuestas, 65,2\%).

- Sobre la capacitación en "Habilidades de comunicación en entornos presenciales y virtuales", en junio de 2020 (246 respuestas, un $82,6 \%$ de los que lo realizaron).

- Sobre las Jornadas de oposiciones (125 respuestas, 35,7\% de los participantes)

Para la recogida de la información se incluyeron afirmaciones o ítems a valorar mediante una escala tipo Likert de 5 puntos o con puntuaciones entre 1-5 o 1-10 (según el instrumento). Adicionalmente se incluyeron preguntas abiertas para recoger impresiones y profundizar en el estudio. Los datos se analizaron en SPSS.

\section{Resultados}

A continuación, se presentan los principales resultados que se desprenden de las acciones llevadas a cabo.

\section{A. Acciones encaminadas a la orientación hacia la profesión docente}

Las mesas redondas de orientación a la función docente son valoradas muy positivamente por los PF y puesta en valor por los propios ponentes, por los importantes beneficios que aportan en términos de orientación hacia el ámbito laboral, y en la que los PF apenas acaban de introducirse. Quizás la valoración es mayor al ser el primer contacto de los PF, con escasa o nula formación docente, con los centros de secundaria.

Las Jornadas de Oposiciones han recibido una valoración media de 8.88 (en una escala de 1 a 10 ), estando muy próximas a ésta las valoraciones de todas las sesiones (de S1 a S5, ver tabla 1).

Tabla 1.

Valoración sesiones de las jornadas oposiciones

\begin{tabular}{|c|c|c|c|c|c|c|c|}
\hline & & VAL.GRAL & S1 & $\mathrm{S} 2$ & $\mathrm{~S} 3$ & $\mathrm{~S} 4$ & S5 \\
\hline \multirow[t]{2}{*}{$\mathrm{N}$} & Vál. & 120 & 122 & 122 & 118 & 121 & 113 \\
\hline & Perd. & 5 & 3 & 3 & 7 & 4 & 12 \\
\hline \multicolumn{2}{|c|}{ Media } & 8,88 & 8,84 & 7,86 & 8,84 & 9,01 & 8,45 \\
\hline \multicolumn{2}{|c|}{ Mediana } & 9,00 & 9,00 & 8,00 & 9,00 & 9,00 & 9,00 \\
\hline \multicolumn{2}{|c|}{ Desv. } & ,922 & 1,188 & 1,815 & 1,365 & 1,158 & 1,653 \\
\hline \multicolumn{2}{|c|}{ Varianza } &, 850 & 1,411 & 3,294 & 1,863 & 1,342 & 2,732 \\
\hline
\end{tabular}

Para analizar el efecto de la asistencia a las Jornadas de Oposiciones se analizan tres variables (conocimientos, intención de presentarse a las oposiciones y confianza en su superación) sobre las cuales se tomaron dos mediciones a la misma muestra. En la diferencia de las tres variables analizadas, la $\mathrm{Z}$ de Kolmogorov-Smirnov tiene un p-valor inferior a 0.05 (tabla 2).

Tabla 2.

Análisis normalidad jornadas oposiciones

\begin{tabular}{crrrrrr} 
& Media & Mediana & Desv. & Varianza & Z (K-S) & P-valor \\
\hline PRE_CONO & 5,03 & 5,00 & 2,355 & 5,547 & 0,102 & 0,003 \\
\hline POST_CONO & 8,14 & 8,00 & 1,255 & 1,576 & 0,192 & 0,000 \\
\hline DIF_CONO & $-3,10$ & $-3,00$ & 2,246 & 5,046 & 0,102 & 0,003 \\
\hline PRE_INTEN & 8,44 & 10,00 & 2,138 & 4,571 & 0,271 & 0,000 \\
\hline POST_INTEN & 8,91 & 10,00 & 1,529 & 2,339 & 0,282 & 0,000 \\
\hline DIF_INTEN & $-0,47$ & 0,00 & 1,280 & 1,638 & 0,356 & 0,000 \\
\hline PRE_CONF & 6,22 & 6,00 & 2,074 & 4,300 & 0,109 & 0,001 \\
\hline POST_CONF & 7,40 & 8,00 & 1,801 & 3,242 & 0,159 & 0,000 \\
\hline DIF_CONF & $-1,18$ & $-1,00$ & 1,858 & 3,453 & 0,143 & 0,000
\end{tabular}

La diferencia de valores no tiene entonces una distribución normal, por lo que aplicamos la técnica no paramétrica Prueba de los rangos con signo de Wilcoxon, para comparar el rango medio de dos muestras relacionadas y determinar si existen diferencias entre ellas. Las hipótesis alternativas o del investigador han sido:

$\mathrm{H}_{1}$ : Los conocimientos de los PF sobre el proceso de las oposiciones son mayores después de asistir a las jornadas

$\mathrm{H}_{2}$ : La intención de presentarse a oposiciones por parte de los PF aumenta después de asistir a las jornadas

$\mathrm{H}_{3}$ : Los $\mathrm{PF}$ sienten más confianza para superar las oposiciones después de asistir a las jornadas

Tabla 3.

Repercusión en los PF de la asistencia a jornadas oposiciones

\begin{tabular}{l|ccc}
\hline & $\begin{array}{c}\text { POST_CONO } \\
- \text { PRE_CONO }\end{array}$ & $\begin{array}{c}\text { POST_INTEN } \\
\text { - PRE_INTEN }\end{array}$ & $\begin{array}{c}\text { POST_CONF - } \\
\text { PRE_CONF }\end{array}$ \\
\hline $\begin{array}{l}Z \text { Prueba de rangos con } \\
\text { signo de Wilcoxon }\end{array}$ & $-8,934^{\mathrm{a}}$ & $-3,933^{\mathrm{a}}$ & $-6,050^{\mathrm{a}}$ \\
\hline Sig. asintótica(bilateral) & 0,000 & 0,000 & 0,000 \\
\hline
\end{tabular}

a. Se basa en rangos negativos.

Como le hemos dado sentido unilateral a las hipótesis, trabajamos con un $\mathrm{p} / 2$. Para las tres variables dicho $\mathrm{p}$-valor es menor que el nivel de significación $(0.05)$ por lo que rechazamos la hipótesis nula, aceptando las alternativas (tabla 3). De esta forma podemos concluir que la asistencia a las jornadas supone un incremento en los conocimientos de los PF sobre el proceso de oposiciones, en la intención de presentarse a oposiciones y en la confianza para su superación con éxito.

\section{B. Capacitación en habilidades de comunicación}

La valoración general que los PF realizan de esta formación para su futuro como docentes es de 8.05. Aunque han sido 6 los grupos en los que se organizaron los PF para recibirla de forma online, no se observan diferencias significativas entre ellos. En la tabla 4 recogemos las puntuaciones medias. Los PF valoran el enfoque que se hace en esta formación hacia las habilidades de comunicación en entornos online, en la que han tenido lugar muchas de sus intervenciones y habida cuenta de una carencia de conocimientos previos al respecto. Las técnicas aprendidas son puestas en práctica por los profesores en formación en varios momentos en los que han debido enfrentarse a hablar en público: en las prácticas en los centros de secundaria (tanto en 
clases presenciales como online) y en la defensa del Trabajo Fin de Máster (TFM) que, dependiendo de la situación sanitaria fueron presenciales u online. Esta habilidad no la hubieran adquirido a partir de un programa de formación tradicional, en el que no se contempla por lo general este tipo de escenarios pero que tan interesantes son en la actualidad y todo indica que serán en un futuro. Los PF muestran la satisfacción con las formadoras tanto en dominio de la materia $(9,07)$, claridad en las explicaciones $(9,15)$ y disposición para aclarar dudas $(9,28)$.

Tabla 4.

Percepción de la utilidad de la formación en habilidades de comunicación en entornos presenciales y virtuales

\begin{tabular}{lcc}
\hline \multicolumn{1}{c}{ (Escala 1-10) } & Media & Desv. \\
\hline $\begin{array}{l}\text { ¿Consideras que las técnicas de comunicación } \\
\text { aprendidas van a resultarte útiles en tu labor docente? }\end{array}$ & 8,18 & 1,645 \\
\hline $\begin{array}{l}\text { ¿Consideras que las técnicas aprendidas te ayudarán a } \\
\text { realizar mejores exposiciones en público en entorno } \\
\text { presencial? }\end{array}$ & 8,21 & 1,550 \\
\hline $\begin{array}{l}\text { ¿Consideras que las técnicas aprendidas te ayudarán a } \\
\text { realizar mejores exposiciones en entorno virtual? }\end{array}$ & 8,16 & 1,625 \\
\hline $\begin{array}{l}\text { ¿Consideras que las técnicas aprendidas serán } \\
\text { aplicables a tu defensa del TFM? }\end{array}$ & 8,15 & 1,386 \\
\hline
\end{tabular}

\section{Capacitación en herramientas docentes digitales}

En el curso 2019/20, cuando fue obligatoria esta formación, participaron $352 \mathrm{PF}$ (finalizándola el 88,1\%). En el curso 2020/21 fue voluntaria, inscribiéndose 265 PF (finalizaron el $45,7 \%$ ). En la tabla 5 se recogen las puntuaciones medias a varios de los aspectos valorados.

Tabla 5.

Valoración de la formación en herramientas docentes digitales

\begin{tabular}{lcccc}
\cline { 2 - 5 } \multicolumn{1}{c}{ (Escala 1-5) } & $\mathrm{n}$ & Media & $\mathrm{n}$ & Media \\
\hline Calidad de los contenidos & 329 & 4,43 & 79 & 4,82 \\
\hline Labores de tutorización & 316 & 4,33 & 76 & 4,74 \\
\hline Gestión de la formación & 325 & 4,13 & 77 & 4,66 \\
\hline Campus virtual & 327 & 4,44 & 79 & 4,78 \\
\hline Cumplimento expectativas & 321 & $96 \%$ & 78 & $98,7 \%$ \\
\hline
\end{tabular}

Los PF valoraron muy positivamente la calidad de los contenidos y la tutorización que recibieron de los mentores. También agradecieron que las labores de matriculación para el curso y la gestión administrativa dentro del mismo fuese muy buena, aspecto importante cuando la comunicación de la universidad con estos participantes se realizaba completamente por medios telemáticos. La plataforma en la que se integró la formación era sencilla e intuitiva, lo que facilitó el aprendizaje por parte de los PF. La formación recibida en el uso de herramientas docentes digitales cumplió ampliamente las expectativas de los PF. En las preguntas abiertas, los PF valoraron muy positivamente la posibilidad de realizar la formación a su ritmo, pero reclamaron que los contenidos estuvieran disponibles desde el principio. También muy valorado que el material incluyera videos, fundamentales para comprender el funcionamiento de plataformas de aprendizaje y destacando que aportan flexibilidad. Asimismo, coincidían en reclamar la descarga de vídeos para posteriores consultas. Todas estas cuestiones se solventaron en la edición del curso 2020/21, motivo que pueden explicar mejores puntuaciones. Los PF destacaron la conveniencia de que esta formación sea obligatoria en la preparación inicial del profesorado, realizarse antes de la incorporación a los centros, tener una mayor duración e incluir muchos ejercicios prácticos. Asimismo, indicaron la necesidad de ampliar a otros entornos y aplicaciones.

\section{DisCUSIÓN}

Son varios los factores que inciden positivamente sobre el rendimiento de los alumnos. En nuestro país, según el informe de Núñez et al. (2020), los dos principales problemas están en la selección y formación inicial de los docentes y en el nivel de autonomía y aprendizaje en el aula. Aunque en la formación que se imparte a PF en el Máster ya se venía trabajando con algunas acciones complementarias, la pandemia vino a reforzar algo: la necesidad de desarrollar nuevas habilidades para entornos cambiantes. Con una adecuada formación en herramientas para el seguimiento online del alumnado, la incorporación de los PF a los centros educativos fue muy positiva en el periodo de marzo a mayo de 2020. Gracias a la formación recibida, PF y profesores de secundaria, se asociaron, proporcionando los primeros ayuda en la planificación e impartición de lecciones. Los PF se convirtieron no sólo en una fuerza auxiliar significativa para los tutores profesionales de los centros educativos, sino que estos roles a veces se han invertido. Los PF han diseñado actividades y desarrollado contenidos, que han podido utilizar los profesores en clase, tutores sometidos en ese momento de confinamiento a una sobrecarga importante de trabajo. Por ejemplo, construyeron salas de escape, crearon videos y diseñaron cuestionarios para su uso en entornos online, entre otro contenido. En la línea de lo que señala DonitsaSchmidt y Ramot (2020), aunque esta relación se ha realizado a través de videoconferencias, se observa un impacto significativo en el aprendizaje profesional de los PF, su identidad docente y su autoeficacia como futuros maestros. Otro efecto de esta situación ha sido la creciente cooperación entre los centros educativos de secundaria y el máster de formación del profesorado.

El próximo desafío es fortalecer y mantener los logros alcanzados con esta formación complementaria. Los programas de formación docente tienen el deber de dotar a los futuros profesores de los conocimientos, las herramientas y la resiliencia que les ayuden a afrontar de manera eficaz los próximos retos educativos, como los que se enfrentaron durante la pandemia de Covid-19 (Donitsa-Schmidt y Ramot, 2020). Durante ese período, se puso de manifiesto la falta de formación de los docentes en activo en el uso de las TIC, por lo que algunos autores propusieron la necesidad de cursos dirigidos a los docentes en uso y manejo de las TIC y la teleformación (Manzano-Sánchez et al., 2021). En esta línea se ha trabajado con la formación en herramientas digitales, la cual se amplió y mejoró de un curso a otro. Coincidimos, asimismo, con Cifuentes-Faura (2020), cuando señala que en los entornos online se debe mantener en todo momento la motivación del alumno para el estudio, la asistencia a clases virtuales y la realización de tareas. Las acciones relacionadas con la formación en herramienta digitales para el seguimiento online del alumnado y la formación en técnicas de comunicación han permitido también trabajar en este sentido. La capacitación en herramientas de comunicación ha sido de gran utilidad para los $\mathrm{PF}$, no sólo en las circunstancias en las que se encontraban ante una docencia no presencial en los centros, sino en su futura experiencia como profesores, por lo que se recomienda su inclusión en los programas formativos. Por otro lado, en momentos de auge en la contratación de docentes, las Jornadas de Oposiciones dentro de la orientación laboral han constituido una de las acciones con mayor acogida. El nivel de asistencia 
de los PF y el feedback recibido son claras evidencias de la necesidad de llevar a cabo estas iniciativas. Comparando las percepciones previas y posteriores a las jornadas, se observaron incrementos significativos en cuanto al conocimiento adquirido sobre las oposiciones, la intención de presentarse a las mismas y la confianza percibida para superarlas. Como concluyen SeinEchaluce et al. (2020) la innovación educativa puede diseñarse globalmente, aplicarse localmente y trasladarse a otros contextos. Es por ello que, aunque el diseño y aplicación de una innovación educativa está condicionada por muchos factores, consideramos posible que estas medidas sirvan de inspiración en otros másteres de formación del profesorado.

\section{CONCLUSIONES}

Consideramos interesante contar con experiencias significativas que ayuden a desarrollar nuevas iniciativas de mejora de la competencia docente del profesorado en su fase de formación inicial. En este sentido, es necesario reflexionar sobre los nuevos contextos, los cambios acaecidos en el entorno y las implicaciones que pueden tener en la adquisición de competencias. Con las acciones formativas que se venían desarrollando en el Máster y las nuevas puestas en marcha con motivo del nuevo escenario educativo, podemos concluir que la formación de los PF ha salido reforzada de este periodo. Lo que comenzó como un aprendizaje de emergencia en una situación de crisis, está evolucionando hacia un aprendizaje de calidad bien planificado para los futuros docentes. Este es el camino por el que hemos constatado que hay que continuar para reforzar las competencias, habilidades y, en general, la formación inicial de nuestros futuros profesores.

\section{REFERENCIAS}

Camus Ferri, M.M., Iglesias Martínez, M.J. y Lozano Cabezas, I. (2019). Un estudio cualitativo sobre la competencia didáctica comunicativa de los docentes en formación. Enseñanza y Teaching, $37 \quad$ (1), 83-101. https://doi.org/10.14201/et201937183101

Cifuentes-Faura, J. (2020). Docencia online y Covid-19: la necesidad de reinventarse. Revista de Estilos de Aprendizaje, 13 (Especial), 115-127. Recuperado de http://revistaestilosdeaprendizaje.com/article/view/2149

Donitsa-Schmidt, S. y Ramot, R. (2020). Opportunities and challenges: teacher education in Israel in the Covid-19 pandemic. Journal of Education for Teaching, 46(4), 586595, doi:10.1080/02607476.2020.1799708

Engen, B. (2019). Understanding social and cultural aspects of teachers' digital competencies. Comunicar, 61, 9-19. https://doi.org/10.3916/C61-2019-01

Fidalgo-Blanco, A., Sein-Echaluce, M.L. y García-Peñalvo, F.J. (2019). Tendencias de innovación educativa con Moodle: llevando el cambio metodológico al aula. Zenodo. http://doi.org/10.5281/zenodo.3381594

López Rupérez, F. y García García, I. (2021). El profesorado de educación secundaria a la luz de PISA 2018Implicaciones para la política educativa española. Madrid: Universidad Camilo José Cela. URI: http://hdl.handle.net/20.500.12020/925

Manso, J. y Martín, E. (2014). Valoración del Máster de Formación del Profesorado de Educación Secundaria: estudios de casos en dos universidades. Revista de
Educación, 364, 145-169. doi: 10.4438/1988-592X-RE2014-364-258

Manzano-Sánchez, D., Valero Valenzuela, A., y HortigüelaAlcalá, D. (2021). Sistema Educativo y actuación ante la pandemia de la COVID-19: opinión y perspectivas de mejora según los docentes. Revista Española de Educación Comparada, (38), 112-128. https://doi.org/10.5944/reec.38.2021.28771

Martínez-Garcés, J. y Garcés-Fuenmayor, J. (2020). Competencias digitales docentes y el reto de la educación virtual derivado de la covid-19. Educación y Humanismo, 22(39),

1-16. https://doi.org/10.17081/eduhum.22.39.4114

Molina-Pérez, J. y Pulido-Montes, C. (2021). COVID-19 y digitalización "improvisada" en educación secundaria: Tensiones emocionales e identidad profesional cuestionada. Revista Internacional de Educación para la Justicia Social, 10(1), 181-196. https://doi.org/10.15366/riejs2021.10.1.011

Muñoz-Fernández, G. A., Rodríguez-Gutiérrez, P., y LuqueVílchez, M. (2019). La formación inicial del profesorado de Educación Secundaria en España: perfil y motivaciones del futuro docente. Educación XX1, 22(1). https://doi.org/10.5944/educxx1.20007

Núñez, C. E. (coordinadora) y otros (2020).Sistema educativo, formación de capital humano, ciencia e investigación tras la COVID-19.FEDEA Policy Papers no. 2020-15. Madrid. Recuperado de https://www.fedea.net/sistemaeducativo-formacion-de-capital-humano-ciencia-einvestigacion-tras-la-covid-19/

Ramos-Huenteo, García-Vásquez, Olea-González, Lobos-Peña y Sáez-Delgado (2020). Percepción docente respecto al trabajo pedagógico durante la COVID-19. CienciAmérica, 9(2), 34-353. http://dx.doi.org/10.33210/ca.v9i2.325

Romero-García C, Buzón-García O, de Paz-Lugo P. (2020). Improving Future Teachers' Digital Competence Using Active Methodologies. Sustainability, 12(18), 7798. https://doi.org/10.3390/su12187798

Sarceda-Gorgoso, C., Santos González, M.C. y Rego-Agraso, L. (2020). Las competencias docentes en la formación inicial del profesorado de Educación Secundaria. Profesorado, Revista de Currículum y Formación del Profesorado, 4 (3), 401-424. https://doi.org/10.30827/profesorado.v24i3.8260

Sein-Echaluce M.L., Fidalgo-Blanco Á., García-Peñalvo F.J., Balbín A.M. (2020). Global Impact of Local Educational Innovation. En: Zaphiris P., Ioannou A. (eds) Learning and Collaboration Technologies. Designing, Developing and Deploying Learning Experiences. HCII 2020. Lecture Notes in Computer Science, vol 12205. Springer, Cham. https://doi.org/10.1007/978-3-030-50513-4_39

Serrano, R. y Pontes, A. (2015). Nivel de desarrollo de las competencias y objetivos generales del Máster Formación del Profesorado de Enseñanza Secundaria. Perfiles Educativos, 37 (150), 39-55. Recuperado de: http://www.scielo.org.mx/pdf/peredu/v37n150/v37n150a 3.pdf 